\title{
Defective activation of neutrophils after splenectomy
}

\author{
PN FOSTER, RP BOLTON, KL COTTER, MS LOSOWSKY \\ From the University Department of Medicine, St James's University Hospital, Leeds
}

SUMMARY Neutrophil chemotaxis and phagocytosis in the presence of serum from 20 patients who had undergone splenectomy and from 15 healthy volunteers was studied. The mean distance migrated by normal neutrophils in the presence of serum from the patients after splenectomy was significantly less than that when normal serum was used $(p<0.005)$. The percentage of neutrophils phagocytosing a yeast was also significantly reduced in the presence of serum from patients after splenectomy $(p<0.02)$. In addition, when neutrophils from these patients were studied both chemotaxis and phagocytosis were enhanced in normal compared with autologous serum $(p<0.05)$.

Fulminant bacterial infection is an uncommon but potentially dangerous complication of splenectomy. Why some patients develop overwhelming sepsis is not completely understood. Neutrophils have an important role in the defence of the host against bacterial infection, but little attention has been paid to the function of these cells after splenectomy, though it has been suggested that the serum of splenectomised patients may lack certain factors that are necessary for normal neutrophil activity. ${ }^{12}$ It is unclear, however, whether these deficiencies lead to substantial impairment of neutrophil function.

We chose to study two aspects of neutrophil function-namely, chemotaxis and phagocytosis in patients who had undergone splenectomy after trauma or for benign conditions - to try to determine the effects of splenectomy rather than those of associated disease on neutrophil function.

\section{Patients and methods}

We studied 20 otherwise healthy patients (13 men, seven women) aged 14-74 (mean 40-3) years who had undergone splenectomy between one and 40 (mean 5.9) years previously. The reasons for splenectomy were: trauma (nine external, six peroperative), hereditary spherocytosis (two), splenic cysts (two), and idiopathic thrombocytopenic purpura (one). Of the six patients whose spleens were removed after peroperative injury, five had under-

Accepted for publication 29 May 1985 gone surgery for benign peptic ulcer disease and one a distal pancreatectomy for chronic pancreatitis. "Pitted" red cell counts were performed for all the patients, who all had counts well above normal $(<$ $4 \%$ ), indicating an absence of effective splenic function. ${ }^{3}$ Fifteen healthy volunteers (seven men, eight women), aged 13-64 (mean 33.3) years served as controls.

We performed two experiments: we measured chemotaxis and phagocytosis by neutrophils from a healthy donor in the presence of serum from the patients who had undergone splenectomy and healthy controls; and in the second experiment we assessed chemotaxis and phagocytosis in the presence of their own or normal serum.

\section{NEUTROPHILS}

Neutrophils were obtained from heparinised venous blood by centrifugation on a Ficol-metrizoate (Lymphoprep; Pharmacia) density gradient and sedimentation in $3 \%$ dextran. Contaminating erythrocytes were lysed by the addition of $0.87 \%$ Trisbuffered ammonium chloride. The remaining pellet of neutrophils was washed in medium (RPMI; Gibco) and suspended at a final concentration of $2 \times 10^{\%}$ $\mathrm{ml}$. The viability of the cells was determined using the exclusion of trypan blue and was always $>95 \%$.

Chemotaxis was measured using a modification of the Boyden method. ${ }^{4}$ Serum activated by zymosan was used as the chemoattractant, and the distance migrated through the millipore filter (pore size $3 \mu$ ) was determined using the leading front method. Each test was performed in triplicate. 
Phagocytosis was assayed by incubating neutrophils with killed Candida albicans for 30 minutes at $37^{\circ} \mathrm{C}$. A slide and smear preparation stained with Giemsa was made. Two hundred neutrophils were examined microscopically and the number containing intracellular $C$ albicans counted. Each test was performed in duplicate.

Analysis was performed using Student's $t$ test and Wilcoxon's signed rank sum test.

\section{Results}

Fig. 1 shows the distance migrated by neutrophils from a healthy donor in the presence of serum from the 15 healthy controls and 20 splenectomised patients. The mean distance migrated by normal neutrophils in the presence of serum from splenectomised patients was significantly less than that when normal serum was used (71.9 (SD 6.6) $\mu \nu$ $78.2(3.7) \mu ; \mathrm{p}<0.005)$. The distance migrated in the presence of serum from six of the splenectomised subjects was below our limit of normal $(70 \mu)$ and just within the lower limit in a further five subjects.

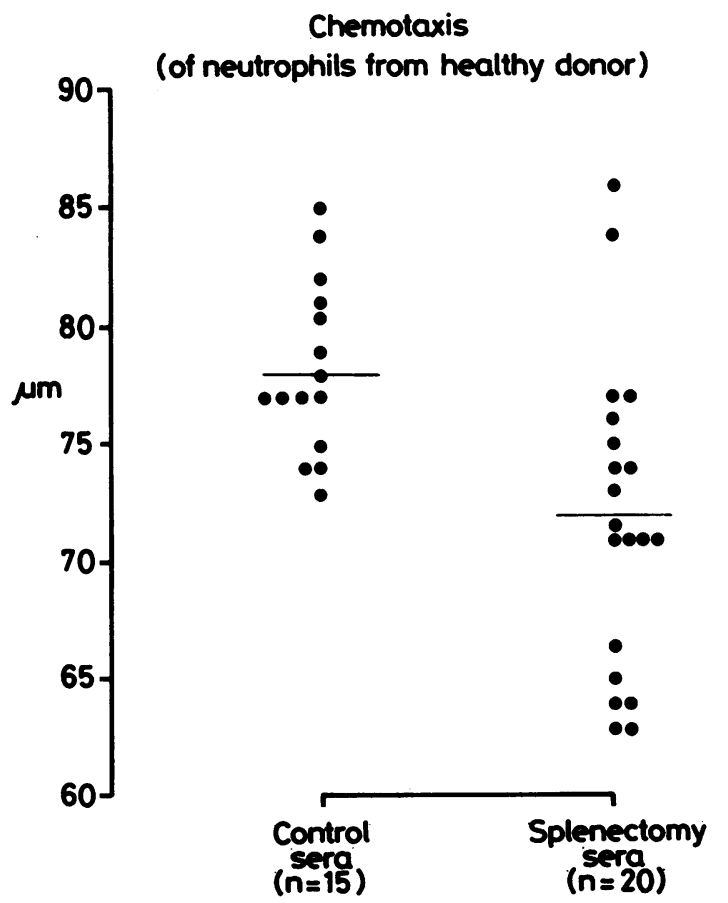

Fig. 1 Chemotaxis of normal neutrophils towards normal serum activated by zymosan and towards serum from splenectomised patients ( $\mu \mathrm{m}=$ distance migrated by leading front of neutrophils through filter).
Fig. 2 shows the percentage of neutrophils from a healthy donor containing intracellular Candida in the presence of serum from the splenectomised patients and controls. The proportion of neutrophils phagocytosing the yeast was abnormally low (normal range $37-56 \%$ ) in the presence of serum from six of the splenectomised patients; the mean was significantly lower than that for normal subjects $(40.2(8.6) \% \vee 46.6(4.6) \%$; $p<0.02)$.

Fig. 3 illustrates the results of the experiment in which chemotaxis and phagocytosis by neutrophils from the splenectomised subjects were assessed in the presence of, firstly, their own serum, and, secondly, normal serum. Both functions were enhanced in the presence of normal serum compared with autologous serum in six of the seven subjects studied. Each result was significant $(p<0.05)$.

\section{Discussion}

We found that neutrophils from patients who had undergone splenectomy could function normally but that serum from some patients did not promote normal chemotaxis or phagocytosis. Previous reports of neutrophil function after splenectomy are conflicting, ${ }^{5-10}$ and the differences in the methods used make comparison difficult.

The work of Deitch and $\mathrm{O}^{\prime} \mathrm{Neal}^{6}$ is most comparzo able to our own. Using neat serum, rather than? serum activated by zymosan, as the chemoattractant

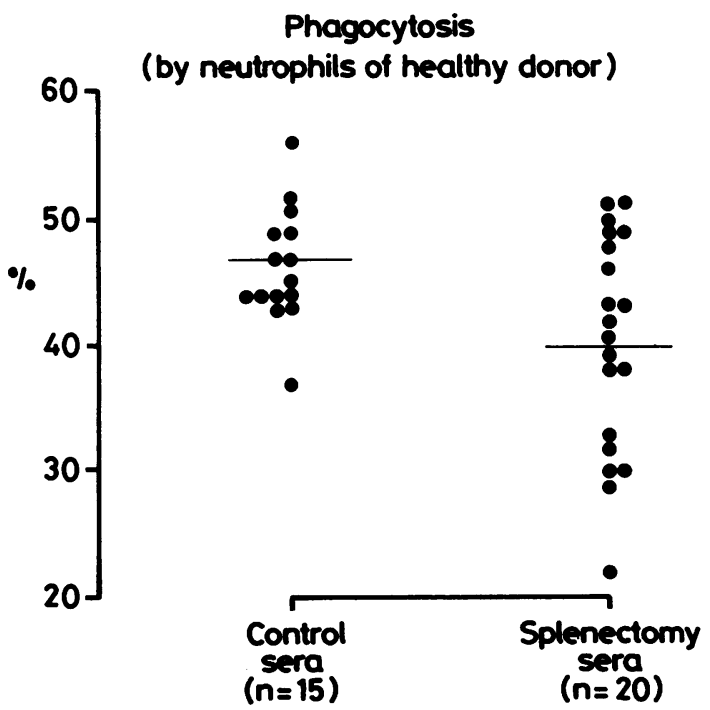

Fig. 2 Phagocytosis of killed Candida albicans by normal neutrophils in presence of normal serum and serum from splenectomised patients. $(\%=$ percentage of 200 cells phagocytosing at least one yeast.) 
Neutrophils from splenectomised patients $(n=7)$
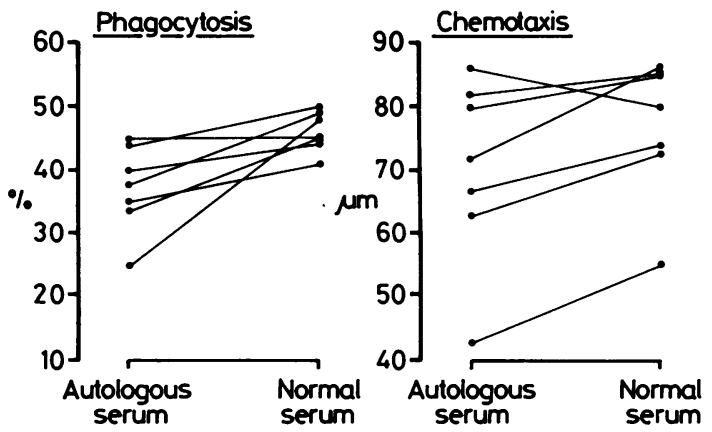

Fig. 3 Phagocytosis and chemotaxis of neutrophils from splenectomised patients in presence of autologous or normal serum. $(\mu \mathrm{m}=$ distance migrated by leading front of neutrophils through filter; \% = percentage of 200 cells phagocytosing at least one yeast.)

and Staphylococcus aureus, rather than $C$ albicans, in the assay of phagocytosis, they concluded that neutrophil chemotaxis, phagocytosis, and intracellular killing of bacteria were normal and that no impairment of serum opsonic or chemotactic activity occurred in patients after splenectomy for trauma. Nevertheless, chemotaxis was appreciably impaired in two of their 14 patients, and the range of the calculated phagocytic index was $0 \cdot 25-1 \cdot 1$ in the presence of serum from splenectomised patients compared with $0.6-1.8$ in the presence of control serum, suggesting that phagocytosis was reduced in the presence of serum from the operated patients, although the difference was not significant.

Using reduction of the dye nitroblue tetrazolium as an index of neutrophil function, Falcao et al found significant abnormalities after splenectomy but Hauser et $\mathbf{a l}^{\mathbf{8}}$ reported no significant difference. Cooper et $a l^{10}$ found no difference in the chemoluminescence generated during phagocytosis or uptake of radiolabelled $S$ aureus in assays comparing serum from splenectomised patients with that from controls.

We found that serum from some splenectomised patients did not promote normal phagocytosis or chemotaxis by neutrophils, and so the question remains whether the serum lacked certain factors or contained inhibitors. Our experiments did not address this problem. Deficiencies of a variety of substances that may be relevant to normal neutrophil function, however, have been reported in patients after splenectomy.

The serum concentration of the tetrapeptide tuftsin is reduced after splenectomy. ${ }^{1}$ Using an assay of phagocytic function similar to our own, Fridkin et $a l^{12}$ showed that tuftsin greatly enhances neutrophil phagocytosis; thus deficiency of this peptide could explain our finding of impaired neutrophil phagocytosis after splenectomy. The role of tuftsin as a chemoattractant is less clear. Although tuftsin can stimulate the random migration of dog polymorphs in glass capillary tubes, ${ }^{13}$ it does not possess chemotactic activity for human neutrophils in micropore filter chambers. ${ }^{14}$

Complement is important in the generation of chemotactic factors. Some workers have found that the concentration of properdin and the activity of the alternative pathway of complement are reduced after splenectomy, ${ }^{15-17}$ but others have disagreed..$^{18-20}$ In our study the alternative pathway of complement was deliberately activated by the addition of zymosan to the serum; this important difference in method might explain the disparity between our results and those of Deitch and O'Neal, ${ }^{6}$ who used untreated serum as the chemoattractant.

Finally, there is evidence from studies on animals that the concentration of circulating fibronectin, a large glycoprotein that acts as an opsonin, falls after splenectomy, ${ }^{21}$ and serum depleted of opsonic fibronectin has a reduced ability to support phagocytosis of bacteria by neutrophils. ${ }^{22}$

In conclusion, our work provides further evidence that the serum from splenectomised patients may not promote normal phagocytosis and chemotaxis by neutrophils. In a proportion of patients this results in significant impairment of neutrophil function, which in turn might contribute to the increased risk of fulminant bacterial infection after splenectomy.

\section{References}

' Eichnor ER. Splenic function: normal, too much and too little. Am J Med 1979;66:311-20.

2 Bullen AW, Losowsky MS. Consequences of impaired splenic function. Clin Sci 1979;57:129-37.

${ }^{3}$ Pearson HA, Johnston MT, Smith KA, Touloukian RJ. The born-again spleen. Return of splenic function after splenectomy for trauma. N Engl J Med 1978;298:1389-92.

${ }^{4}$ Frei PC, Balsero M, Ochsner M. Chemotaxis of human polymorphonuclears in vitro. II. Technical study. J Immunol Methods 1974;5:373-86.

s Fliedner von V, Salvatori V, Higby DJ, Stutzman L, Park BH. Polymorphonuclear neutrophil function in malignant lymphomas and effects of splenectomy. Cancer 1980;45:469-75.

- Deitch EA, O'Neal B. Neutrophil function in adults after traumatic splenectomy.J Surg Res 1982;33:98-102.

' Falcao RP, Voltarelli JL, Bottura C. The possible role of the spleen in the reduction of nitroblue tetrazolium by neutrophils. Acta Haematol 1982;68:89-95.

${ }^{8}$ Hauser GJ, Zakuth V, Spirer Z. Normal reduction of nitroblue tetrazolium by neutrophils of splenectomized individuals. Acta Haematol 1983; 70:142-3.

9 Constantopolous A, Najjar VA, Wish JB, Necheles JH, Stolbach LL. Defective phagocytosis due to tuftsin deficiency in splenectomized subjects. Am J Dis Child 1973;125:663-5. 
${ }^{10}$ Cooper MR, Dechatelet LR, Shirley PS, Cooper RM. Does tuftsin alter phagocytosis by human polymorphonuclear neutrophils? Inflammation 1982;6:103-12.

" Spirer Z, Zakuth V, Diaments S, et al. Decreased tuftsin concentrations in patients who have undergone splenectomy. $\mathrm{Br}$ Med J 1977;ii: 1574-6.

${ }^{12}$ Fridkin M, Stabinsky Y, Zakuth V, Spirer Z. Tuftsin and some analogs; synthesis and interaction with human polymorphonuclear leucocytes. Biochim Biophys Acta 1977; 496: 203-11.

${ }^{13}$ Nishioka K, Satoh PS, Constantopolous A, Najjar V. The chemical synthesis of the phagocytosis stimulating tetrapeptide tuftsin and its biological properties. Biochim Biophys Acta 1973;310:230-7.

${ }^{14}$ Goetzl EJ. Neutrophil chemotactic inhibitors. Ann NY Acad Sci 1975;256:210-21.

is Carlisle HN, Saslaw S. Properdin levels in splenectomized persons. Proc Soc Exp Biol Med 1959;102:150-4.

${ }^{16}$ Polhill RB, Johnston RB. Diminished alternative complement pathway activity after splenectomy. Pediatr Res 1975;9:333.

17 Corry JM, Polhill RB, Edmonds SR, Johnston RB. Activity of the alternative pathway complement after splenectomy: comparison to activity in sickle cell disease and hypogamma- globulinaemia. J Pediatr 1979;95:964-9.

${ }^{18}$ Winkelstein JA, Lambert GH. Pneumococcal serum opsonizing activity in splenectomized children. J Pediatr 1975;87:430-3.

${ }^{19}$ Ciuttis A, Polley MJ, Metakis LJ, Peterson MJ. Immunologic defect of the alternative pathway of complement activation post-splenectomy: a possible relation between splenectomy and infection. J Natl Med Assoc 1978;70:667-70.

${ }^{20}$ Lanng-Neilsen J, Buskjaer L, Lamm U, Solling J, Ellegard J. Complement studies in splenectomized patients. Scand J Haematol 1983;30:194-200.

${ }^{21}$ Hashimoto T, Mahour HG, Church JA, Lipsey AI. Plasma fibronectin levels after splenectomy and splenic autoimplantation in rats with and without dietary acorbic acid supplementation. J Pediatr Surg 1983;18:805-9.

${ }^{22}$ Lanser ME, Saba TM. Fibronectin as a co-factor necessary for optimal granulocyte phagocytosis of Staphylococcus aureus. $J$ Reticuloendothel Soc 1981;30:415-23.

Requests for reprints to: Dr RP Bolton, Department of Medicine, St James's University Hospital, Leeds LS9 7TF, England. 